\title{
Momentum Constant Modulus Blind Equalization Algorithm Based on Global Artificial Fish Swarm Optimization Algorithm
}

\author{
Chuan $\mathrm{HE}^{1}$, Yecai GUO ${ }^{2, \mathrm{a}^{*}}$, Hui WANG ${ }^{2}$ and Lihua WANG ${ }^{2}$ \\ ${ }^{1}$ College of Atmospheric Physics, Nanjing University of Information Science and Technology, \\ Nanjing 210044, China \\ ${ }^{2}$ Jiangsu Collaborative Innovation Center on Atmospheric Environment and Equipment Technology \\ (CICAEET), Nanjing 210044, China \\ ${ }^{{ }^{\star}}$ email: guo-yecai@163.com
}

Keywords: Momentum constant blind equalization; Global artificial fish swarm algorithm; Convergence speed

\begin{abstract}
In order to overcome local convergence of constant modulus blind equalization algorithm (CMA), the momentum constant modulus blind equalization algorithm(MCMA) based on global artificial fish swarm optimization algorithm(GAFSA-MCMA) is proposed. In this proposed algorithm, on the basis of making full use of the global artificial fish swarm algorithm(AFSA) with the fast convergence and global search ability, the position vector of the artificial fish is optimized and the global optimal position vector is used as the initial optimal weight vector of the MCMA. Compared with the CMA and MCMA, the proposed algorithm has fastest convergence rate and minimum mean square error(MSE).
\end{abstract}

\section{Introduction}

In the underwater communication system, the random characteristics of underwater acoustic channel are time varying and frequency varying, so the underwater acoustics signal is subjected to multipath fading and limited bandwidth in the transmission process. In order to eliminate these effects and improve the performance of the communication system, a blind adaptive equalization technique is used in the receiving end. In the existing blind equalization algorithms, the convergence rate and the steady mean square error(MSE) of the constant modulus blind equalization algorithm (CMA) are determined by the step-size factor[1][2]. When the step-size increases, the CMA has faster convergence rate and larger mean square error. However, the momentum constant modulus blind equalization algorithm(MCMA) can effectively improve the convergence speed without affecting the residual MSE[3]. So, it is necessary for using momentum alorithm to accelerate the convergence rate of the CMA. The global artificial fish swarm algorithm(GAFSA) is an efficient intelligence algorithm with global search ability and can overcome the low accuracy, slow convergence rate, and high complexity of the AFSA[4][5].

In this paper, based on the advantages of the MCMA and GAFSA, the momentum constant modulus blind equalization algorithm based on global artificial fish swarm optimization algorithm (GAFSA -MCMA) is proposed to overcome the defects of the CMA.

\section{Momentum Constant Modulus Blind Equalization Algorithm (MCMA)}

Blind equalization algorithm is a method, which uses the statistical property of the received signals, and it has no training sequences and can make output signals equal to input signals as much as possible. In the momentum constant modulus blind equalization algorithm, $\boldsymbol{a}(k)$ is a zero mean independent identically distributed transmitted signal, $\boldsymbol{c}(k)$ denotes channel impulse response, and $\boldsymbol{n}(k)$ is an additive white Gaussian noise vector, as well as $\boldsymbol{y}(k)$ represents the received signal to the equalizer. Moreover, $f(k)$ is the equalizer weight vector and $z(k)$ is the equalizer output signal.

Their relationships are given as follows: 


$$
\begin{aligned}
& \boldsymbol{y}(k)=\boldsymbol{c}^{\mathrm{T}} \boldsymbol{a}(k)+\boldsymbol{n}(k) \\
& z(k)=\boldsymbol{f}^{\mathrm{T}}(k) \boldsymbol{y}(k)=\boldsymbol{y}^{\mathrm{T}}(k) \boldsymbol{f}(k)
\end{aligned}
$$

So, the error term for the MCMA is defined as

$$
e(k)=|z(k)|^{2}-R^{2}
$$

where $R^{2}$ is the modulus value of the transmitted signals, and $R^{2}=E\left\{|\boldsymbol{a}(k)|^{4}\right\} / E\left\{|\boldsymbol{a}(k)|^{2}\right\}$. In the MCMA, the cost function is defined as

$J=E\left[e^{2}(k)\right]$

The weight vector updating equation of the MCMA is written as

$$
\boldsymbol{f}(k+1)=\boldsymbol{f}(k)-\mu e(k) z(k) \boldsymbol{y}^{*}(k)+\alpha[\boldsymbol{f}(k)-\boldsymbol{f}(k-1)]
$$

where $\mu$ is an iterative step-size and $\alpha$ is a momentum factor.

\section{Global Artificial Fish Swarm Algorithm (GAFSA)}

The GAFSA is an efficient intelligence algorithm, which can imitate fish swarm's behaviors including preying, swarming, and following end collision to achieve the global optimal soulation.

Preying behavior. Let $\boldsymbol{X}_{i}$ be the current position vector of the global artificial fish and a position vector $\boldsymbol{X}_{i}$ in current visual distance is selected randomly, we use $Y\left(\boldsymbol{X}_{i}\right)$ to denote the fitness function of the global artificial fish. If the fitness function value $Y\left(\boldsymbol{X}_{j}\right)>Y\left(\boldsymbol{X}_{i}\right)$, then the global artificial fish goes a step from the position $\boldsymbol{X}_{i}$ to position $\boldsymbol{X}_{j}$ according to Eq.6, otherwise, the global artificial fish selects another position vector $\boldsymbol{X}_{i}$ again and judges whether it is reach the forward movement condition. If it can't reach the forward movement condition, it randomly moves a step forward according to Eq.7.

$$
\begin{aligned}
& \boldsymbol{X}_{i+1}=\boldsymbol{X}_{i}+\frac{\left(\boldsymbol{X}_{j}-\boldsymbol{X}_{i}\right)+\left(\boldsymbol{X}_{\text {best }}-\boldsymbol{X}_{i}\right)}{\left\|\left(\boldsymbol{X}_{j}-\boldsymbol{X}_{i}\right)+\left(\boldsymbol{X}_{\text {best }}-\boldsymbol{X}_{i}\right)\right\|} \cdot \text { step } \cdot \operatorname{rand}(0,1) \\
& \boldsymbol{X}_{i+1}=\boldsymbol{X}_{i}+\mathrm{V} \cdot \operatorname{rand}(0,1)
\end{aligned}
$$

where rand $(0,1)$ means random number within 0 to 1 , step is a step-size, $\mathrm{V}$ is a visual range, and $\boldsymbol{X}_{\text {best }}$ is the best position vector.

Swarming behavior. Assume that the current position vector $\boldsymbol{X}_{i}$ of the global artificial fish and the center position vector $\boldsymbol{X}_{c}$ in the current visual range and the number of the companions of the artificial fishes can be searched. If the fitness function value $Y\left(\boldsymbol{X}_{c}\right)>Y\left(\boldsymbol{X}_{i}\right)$, the center position has more food and artificial fishes aren't too crowded. Then the artificial fish goes a step towards the center position according to Eq.8.

$$
\boldsymbol{X}_{i+1}=\boldsymbol{X}_{i}+\frac{\left(\boldsymbol{X}_{c}-\boldsymbol{X}_{i}\right)+\left(\boldsymbol{X}_{\text {best }}-\boldsymbol{X}_{i}\right)}{\left\|\left(\boldsymbol{X}_{c}-\boldsymbol{X}_{i}\right)+\left(\boldsymbol{X}_{\text {best }}-\boldsymbol{X}_{i}\right)\right\|} \cdot \text { step } \cdot \operatorname{rand}(0,1)
$$

Following behavior. Assume that $\boldsymbol{X}_{\max }$ denotes the position vector corresponding to the maximum fitness function value in current visual distance. If the number of companions of the artificial fishes in the visual distance meets the condition for $N_{v} / N>\delta$, where $\delta$ denotes congestion degree and $N_{v}$ is the number of companions in the visual distance. If the fitness function value $Y\left(\boldsymbol{X}_{\max }\right)>Y\left(\boldsymbol{X}_{i}\right)$, the artificial fish goes a step towards position vector $\boldsymbol{X}_{\max }$ according to Eq.9.

$$
\boldsymbol{X}_{i+1}=\boldsymbol{X}_{i}+\frac{\left(\boldsymbol{X}_{\max }-\boldsymbol{X}_{i}\right)+\left(\boldsymbol{X}_{\text {best }}-\boldsymbol{X}_{i}\right)}{\left\|\left(\boldsymbol{X}_{\max }-\boldsymbol{X}_{i}\right)+\left(\boldsymbol{X}_{\text {best }}-\boldsymbol{X}_{i}\right)\right\|} \cdot \text { step } \cdot \operatorname{rand}(0,1)
$$




\section{MCMA Based on GAFSA(GAFSA-MCMA)}

The MCMA based on global artificial fish swarm optimization algorithm is a further optimization of the CMA. By the GAFSA, the initial weight vector of the MCMA is optimized to improve the convergence rate and reduce the MSE. The steps of the proposed algorithm are given as follows:

Step1. Initialization of artificial fish. The initial population of the artificial fish swarm $\boldsymbol{X}=\left[\boldsymbol{X}_{1}, \boldsymbol{X}_{2}, \cdots, \boldsymbol{X}_{\mathrm{N}}\right]$, where $\boldsymbol{X}_{i}$ is the initial position vector of the $i$ th artificial fish. $N$ is the number of artificial fish.

Step2. Determination of fitness function. The GAFSA is designed to find the position vector corresponding to the maximum value of the fitness function, and the position vector is used as the initial optimal weight vector of the equalizer. So, the reciprocal of the cost function is used as the fitness function of the global artificial fish swarm algorithm. The fitness function is defined as $Y\left(\boldsymbol{X}_{i}\right)=1 / J\left(\boldsymbol{f}_{i}\right), \quad i=1,2, \ldots, N$, where $J\left(\boldsymbol{f}_{\boldsymbol{i}}\right)$ is the cost function of the MCMA.

Step3. The calculation of fitness function. The fitness function of each artificial fish is calculated according to step2. The maximum fitness function and its corresponding position vector are recorded in the bulletin board.

Step4. Each artificial fish in the fish swarm carries out following behavior according to Eq.9. If the following behavior doesn't occur, all fishes will carry out swarming behavior according to Eq.8. If swarming behavior is also not succeed, all fishes will carry out preying behavior according to Eq.6 and Eq.7. Each behavior can cause the change of the current position vector.

Step5. The update of the bulletin board. The fitness function of each artificial fish is calculated. The maximum one of them is selected and compared with the previous maximum value of first fitness function recorded in the bulletin board. If the maximum one of them is greater than the previous maximum value recorded in the bulletin board, the bulletin board is updated by the current maximum value and its corresponding to the position vector.

Step6. Determination of the termination condition. Judge whether the iteration is equal to the maximum value. If the condition doesn't hold, the iteration process goes back to step 4, otherwise, the record in the bulletin board is used as the initial optimization weight vector and updated according to Eq.5.

\section{Simulation Analysis}

In the simulations, the transmitted signals were 16QAM signals, the channel $h=[0.9656-0.0906$ 0.05780 .2368 ], SNR(signal to noise ratio) was $20 \mathrm{~dB}$, the number of equalizer weight coefficients $L=11$, the step-size $\mu{ }_{\mathrm{CMA}}=\mu_{\mathrm{MCMA}}=0.00003, \mu_{\text {GAFSA-MCMA }}=0.00001$. The number of AFS $N=30$, the crowding factor $\delta=0.5$. Simulated results were shown in figure 1 .

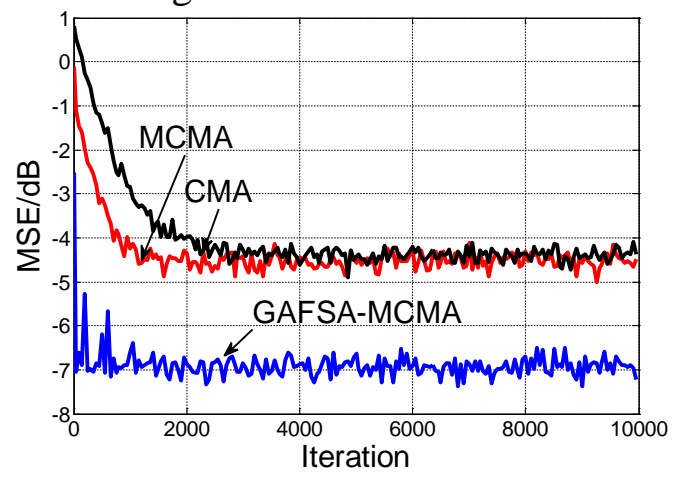

(a) Curves of MSE

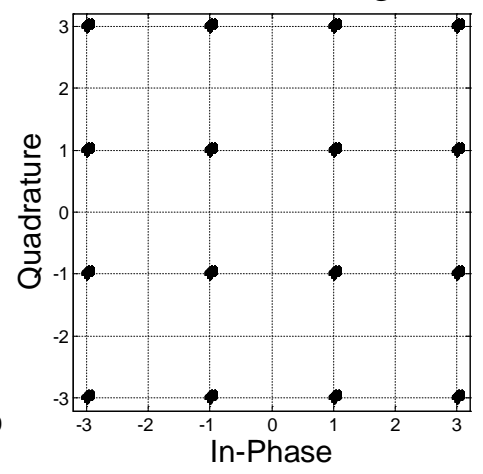

(b) Transmitted constellations

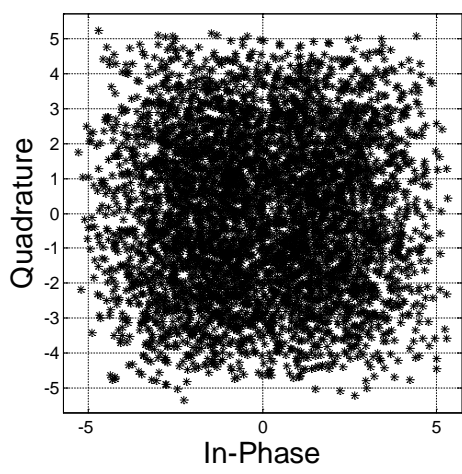

(c) Input of equalizer 


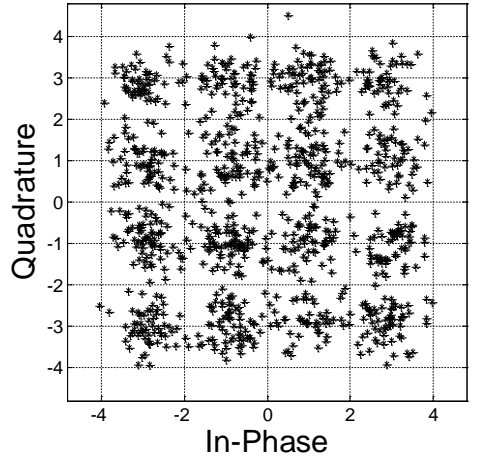

(d) Output of CMA

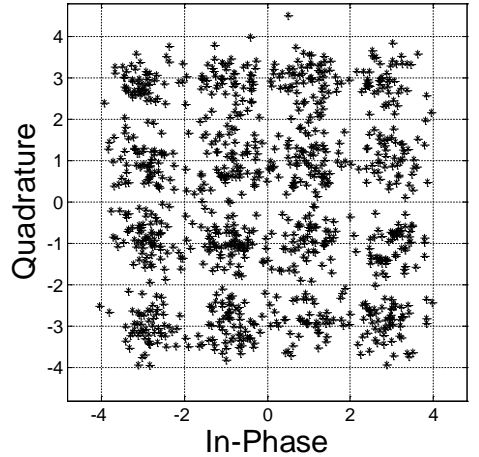

(e) Output of MCMA

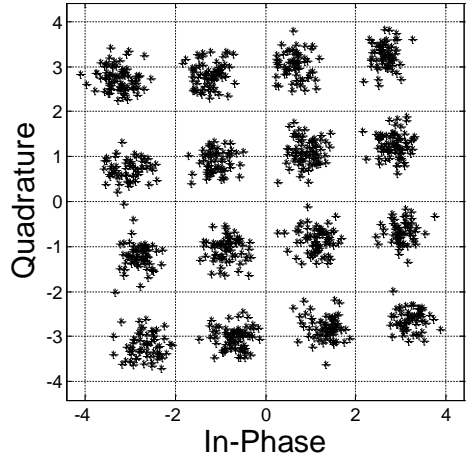

(f) Output of GAFSA-MCMA.

Fig.1. Simulated results

From Figure 1, we can know that the GAFSA-MCMA outperforms the MCMA and CMA in improving the convergence speed and reducing the MSE. The MSE of the GAFSA-MCMA is smallest and its convergence speed is fastest.

\section{Summary}

The global artificial fish swarm algorithm are applied to the momentum constant modulus blind equalization algorithm(MCMA) to optimize further the initial weight vector, the momentum constant modulus blind equalization algorithm based on global artificial fish swarm optimization algorithm(GAFSA-MCMA) is proposed. The simulation results show that the mean square error(MSE) of the GAFSA-MCMA is lowest and its convergence speed is fastest, and its output constellations are more compact and clearer than those of the MCMA and the CMA.

\section{Acknowledgement}

This work is supported by the National Natural Science Foundation of China(51405241, 61371131, 11504176),the Major Project of Nature Science Foundation of Higher Education Institution of Jiangsu Province, China (Grant No.13KJA510001), Jiangsu Scientific Research Achievements in Industrialization Project, China(JHB 2012-9), and Jiangsu Province, the 2015 Annual General University Graduate Students Practice Innovation Program(SJLX15_0398).

\section{References}

[1] Y.Sato. A method of self-recovering equalization for multiple amplitude mod-ulation schemes. IEEE Transactions on Communications, 1975,86(10):1927-1949.

[2] Prof. De. Amin Mohanmed Nassar.New blind equalization technique for constant modulus algorithm(CMA) [J].IEEE International Workshop Technical Committee on Communications Quality and Reliability (CQR),2010,1-6.

[3] Yongguk Kim, Heung-Gyoon Ryu. Coordinate change and SAG MCMA equalizer in the mobile satellite communication system. 15th International Conference on Advanced Communication Technology(ICACT), 2013, 104-108.

[4] Gao Yan-bin, Guan Lian-wu, Wang Ting-jun, et al.. Research on the calibration of fog based on AFSA. IEEE International Conference on Mechatronics and Automation(ICMA), Takamatsu, 2013, 412-417.

[5] Ma Li, Fan Suo-hai. Forex prediction based on SVR optimized by artificial fish swarm algorithm. Fourth Global Congress on Intelligent Systems (GCIS), Hong Kong, 2013:47-52. 